\title{
Pseudo-discordance mimicking low-flow low-gradient aortic stenosis in transcatheter aortic valve replacement patients with severe symptomatic aortic stenosis
}

\author{
Rafael Kuperstein ${ }^{1,2}$, Michael Michlin ${ }^{1,2}$. Israel Barbash ${ }^{1,2}$, Israel Mazin ${ }^{1,2}$, \\ Yafim Brodov ${ }^{1,2,3}$, Paul Fefer ${ }^{1,2}$, Amit Segev ${ }^{1,2}$, Victor Guetta ${ }^{1,2}$, Elad Maor ${ }^{1,2}$, \\ Orly Goiten $^{2,3}$, Michael Arad ${ }^{1,2}$, Micha S. Feinberg ${ }^{1,2}$, Ehud Schwammenthal ${ }^{1,2}$ \\ ${ }^{1}$ The Leviev Heart Center, Sheba Medical Center, Israel \\ ${ }^{2}$ Sackler School of Medicine, Tel Aviv University, Israel \\ ${ }^{3}$ Department of Diagnostic Imaging, Sheba Medical Center, Israel
}

\begin{abstract}
Background: While the combination of a small aortic valve area (AVA) and low mean gradient is frequently labeled 'low-flow low-gradient aortic stenosis (AS)', there are two potential causes for this finding: underestimation of mean gradient and underestimation of AVA.

Methods: In order to investigate the prevalence and causes of discordant echocardiographic findings in symptomatic patients with AS and normal left ventricular (LV) function, we evaluated 72 symptomatic patients with AS and normal LV function by comparing Doppler, invasive, computed tomography $(C T)$ LV outflow tract (LVOT) area, and calcium score ( $\mathrm{CaSc})$.

Results: Thirty-six patients had discordant echocardiographic findings (mean gradient $<40 \mathrm{mmHg}$, $A V A \leq 1 \mathrm{~cm}^{2}$ ). Of those, 19 had discordant invasive measurements (true discordant [TD]) and $17 \mathrm{con}$ cordant (false discordant [FD]): In 12 of the FD the mean gradient was $>30 \mathrm{mmHg}$; technical pitfalls were found in 10 patients (no reliable right parasternal Doppler in 6). LVOT area by echocardiography or CT could not differentiate between concordants and discordants nor between TD and FD ( $p=N S)$. CaSc was similar in concordants and FD $(p=0.3)$, and it was higher in true concordants than in TD ( $p=0.005)$. CaSc positive predictive value for the correct diagnosis of severe AS was $95 \%$ for concordants and $93 \%$ for discordants.

Conclusions: Discordant echocardiographic findings are commonly found in patients with symptomatic AS. Underestimation of the true mean gradient due to technical difficulties is an important cause of these discrepant findings. LVOT area by echocardiography or CT cannot differentiate between TD and FD. In the absence of a reliable and compete multi-window Doppler evaluation, patients should undergo CaSc assessment. (Cardiol J)
\end{abstract}

Key words: aortic stenosis, echocardiography, aortic valve, valvular disease, calcium

\section{Introduction}

Severe aortic stenosis (AS) is defined by an aortic valve (AV) area (AVA) $\leq 1.0 \mathrm{~cm}^{2}$ and a mean gradient $\geq 40 \mathrm{mmHg}[1,2]$. These parameters conflict in a high proportion of patients who present with an AV area $\leq 1 \mathrm{~cm}^{2}$, but a mean gradient $<40 \mathrm{mmHg}$, even when systolic left ventricu-

Address for correspondence: Rafael Kuperstein, MD, The Leviev Heart Center, Sheba Medical Center, Ramat Gan, Israel, ZIP Code: 5265601, tel: +97235302506, fax:+97235307556, e-mail: rafikupe@gmail.com

Received: 4.06.2021 Accepted: 11.08.2021 Early publication date: 23.09.2021

This article is available in open access under Creative Common Attribution-Non-Commercial-No Derivatives 4.0 International (CC BY-NC-ND 4.0) license, allowing to download articles and share them with others as long as they credit the authors and the publisher, but without permission to change them in any way or use them commercially. 
lar (LV) function appears normal [3]. While this discordance is frequently attributed to possible low-flow low-gradient severe AS with preserved LV function, the list of potential sources for discrepancies includes several causes: possible underestimation of Doppler flow velocity and derived mean gradients, echocardiographic underestimation of an elliptical LV outflow tract (LVOT) area [4-6], or small body size with transvalvular flow that is below average, but adequate

Notwithstanding the long list of potential root causes, studies of the syndrome of low flow low gradient severe AS frequently lack a comprehensive approach employing both independent imaging modalities and independent hemodynamic assessment to systematically and reliably rule them out. We therefore sought to investigate the prevalence of a discrepant echocardiographic constellation and the distribution of its individual root causes by comparing Doppler data to invasive hemodynamic data as well as computed-tomographic findings in patients with severe symptomatic AS and normal LV ejection fraction (LVEF).

\section{Methods}

Patients with symptomatic AS and LVEF $>50 \%$, who underwent a complete echocardiographic, computed tomography (CT), and hemodynamic evaluation before transcatheter AV replacement at Sheba Medical Center from 2011 to 2019, were included in this study.

All patients underwent a full cardiac CT scan including quantification of $\mathrm{AV}$ calcification.

The study was authorized be the Sheba Medical Center Helsinki Committee.

\section{Echocardiographic evaluation}

Echocardiographic studies were performed utilizing commercially available machines according to current American Society of Echocardiography guidelines [7]. LVEF was measured using the Simpson method, and valve area was calculated by the continuity equation. Pressure gradients were assessed from continuous wave transvalvular velocity tracings. Stroke volume (SV) was calculated as the product of the time velocity integral (VTI) and cross-sectional area of the LVOT and indexed to body surface area. All studies were performed by specialized echocardiography technologists and reported by a trained echocardiographer. For the purpose of this study, all original recordings were carefully re-evaluated by an experienced echocardiographer (R.K.).

\section{Hemodynamic evaluation}

Right heart catheterization was performed in all patients, recording pulmonary arterial pressure and capillary wedge pressure. Mean pressures were averaged from three cardiac cycles. Cardiac output was determined using the Fick method, and SV was calculated as cardiac output divided by heart rate. Left heart catheterization with retrograde passage of the stenotic AV was performed. Pressure gradients were measured from simultaneous LV and aortic pressure recordings or pullback tracings with electronic alignment of the recorded ventricular and aortic pressure curves, and AVA was calculated using the Gorlin equation.

\section{CT acquisition protocol}

An electrocardiogram (ECG)-gated non-contrast calcium score $(\mathrm{CaSc})$ scan was followed by a contrast-enhanced scan utilizing a 256 -slice scanner (Brilliance iCT, Philips Healthcare; collimation $96 \times 0.625 \mathrm{~mm}$, gantry rotation time of $330 \mathrm{~ms}$, pitch value 0.2 ), with injection of 70 to $85 \mathrm{~mL}$ of a nonionic contrast agent at a flow rate of $3.5 \mathrm{~mL} / \mathrm{s}$ followed by a $30-\mathrm{mL}$ saline chase bolus $(5 \mathrm{~mL} / \mathrm{s})$. Automated peak enhancement detection in the descending aorta was used for timing of the scan, and the data acquisition was automatically initiated at a threshold level of 100 Hounsfield units. Acquisition was performed during an inspiratory breathhold while the ECG was recorded simultaneously to allow retrospective gating of the data.

\section{Calcium score analysis}

Calcium score quantification was performed using dedicated software ("Heartbeat CS", IntelliSpace Portal, version $7 \mathrm{~V}$, Philips) implementing the Agatston method [8-10]. Briefly, lesion-specific scores were calculated as the product of the area of each calcified focus and peak CT Hounsfield units value and summed to obtain a total CaSc carefully excluding nonvalvular calcification of surrounding structures.

Definitions, stepwise analysis, and statistical methods

Patients with discordant echocardiographic findings (mean gradient $<40 \mathrm{mmHg}$ despite AVA $\leq 1 \mathrm{~cm}^{2}$ ), who were confirmed to have a mean gradient $<40 \mathrm{mmHg}$ on invasive measurements, were defined as true discordant (TD). Patients with discordant echocardiographic findings, who had an invasive mean gradient $\geq 40 \mathrm{mmHg}$, were defined as false discordant (FD).

The clinical, echocardiographic, hemodynamic, and $\mathrm{CT}$ characteristics of concordants were 


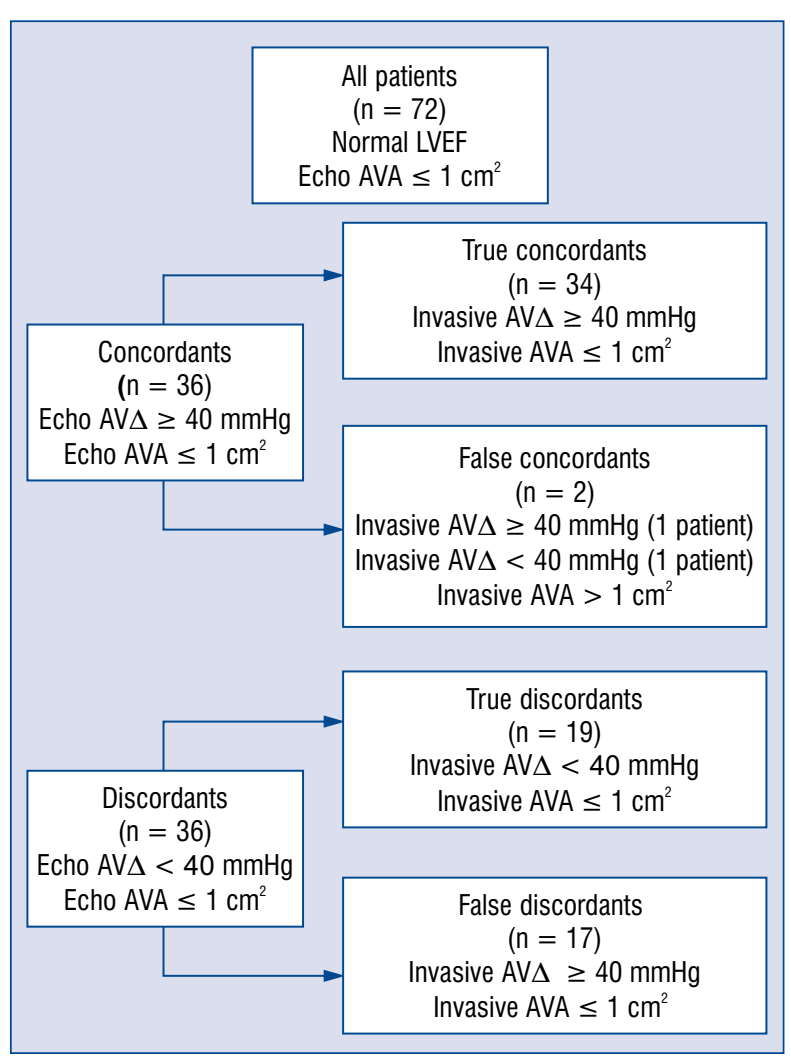

Figure 1. Patient population; AVA - aortic valve area; LVEF - left ventricular ejection fraction.

compared to discordants as a whole, as well as separately to FD and to TD. Finally, FD and TD were compared. Results are presented as mean \pm standard deviation, and the different groups were compared by unpaired two-sample t-test.

Non-parametric data were expressed as percentages and were compared by chi-square test.

The positive predictive value of CaSc for the prediction of severe AS by invasive measurement was calculated for patients with concordant and discordant echocardiographic findings, respectively.

\section{Results}

Seventy-two patients had an echocardiographic AVA $<1.0 \mathrm{~cm}^{2}$; their mean age was $81.6 \pm 6.9$ years, and 41 were females ( $57 \%$ ). The flow chart in Figure 1 shows the patient distribution across the sub-groups defined by the pre-specified criteria. Clinical characteristics, and echocardiographic, invasive hemodynamic, and CT measurements of the whole patient population and the concordant and discordant groups are shown in Table 1.

Thirty-six patients had concordant echocardiographic findings (mean gradient $>40 \mathrm{mmHg}$ and AVA $\leq 1 \mathrm{~cm}^{2}$ ) compatible with high-gradient severe AS (concordants, Table 1), Systolic blood pressure was similar at echocardiography and the invasive evaluation $(139 \pm 29 \mathrm{mmHg}$ and $134 \pm$ $\pm 30 \mathrm{mmHg}$ ). Two patients with high echocardiographic mean gradients had an invasively measured AVA $>1 \mathrm{~cm}^{2}$, and one of them had lower invasive gradient (related to significant pressure recovery) and was hence "false concordant". Consequently, the positive predictive value of concordant findings per echo for concordant findings at cardiac catheterization was $94.4 \%$.

Thirty-six patients showed discordant findings on echocardiography, (mean gradient $<40 \mathrm{mmHg}$ and an AVA $\leq 1 \mathrm{~cm}^{2}$, discordants). Their systolic blood pressure was similar at echocardiography and the invasive evaluation $(137 \pm 27 \mathrm{mmHg}$ and $136 \pm 27 \mathrm{mmHg})$. Overall, when compared to concordants, discordants showed similar clinical characteristics (Table 1) and showed no significant difference in LVOT area. However, discordants had lower mean gradient, larger AVA, lower $\mathrm{CaSc}$, and smaller $\mathrm{SV}$ index with values $\leq 35 \mathrm{~mL} / \mathrm{m}^{2}$ being significantly more prevalent $(\mathrm{p}=0.006)$.

Nineteen of the discordant patients had an invasive hemodynamic mean gradient $<40 \mathrm{mmHg}$ (TD), and 17 discordant patients showed hemodynamic mean gradients $\geq 40 \mathrm{mmHg}$ (FD) (Table 2). Consequently, discordant findings per echocardiography had only a $52.7 \%$ positive predictive value for discordant findings at cardiac catheterization.

True discordants differed from the true concordant mainly in echocardiographic Doppler parameters, with smaller LVOT VTI, smaller AV VTI with lower mean gradients, lower SV indices, and slightly larger AVA (Table 2). Their invasive hemodynamic AVA was similar, their mean gradients were lower, and their $\mathrm{CaSc}$ were lower $(\mathrm{p}=0.005)$; $\mathrm{SV}$ index $\leq 35 \mathrm{~mL} / \mathrm{m}^{2}$ was significantly more prevalent among TD than in TC $(\mathrm{p}=0.01)$.

False discordants differed from TC only in their echocardiographic Doppler parameters with lower LVOT VTI, lower AV VTI, lower mean gradients, slightly larger AVA, and a lower SV index (Table 2). On invasive evaluation, TC and FD had similar AVA, similar mean gradients, and a similar $\mathrm{CaSc}$ indicating similar disease severity (Table 2). $\mathrm{SV}$ index $\leq 35 \mathrm{~mL} / \mathrm{m}^{2}$ was significantly more prevalent among FD than in TC $(\mathrm{p}=0.04)$.

When compared to FD, TD had lower mean echocardiographic gradients and similar AVA and $\mathrm{SV}$ index. On invasive evaluation their gradients were significantly lower, and their AVA was simi- 
Table 1. Baseline clinical, echocardiographic, invasive hemodynamic, and computed tomography characteristics: Concordants vs. discordants.

\begin{tabular}{|c|c|c|c|c|}
\hline & All $(n=72)$ & Concordant $(n=36)$ & Discordant $(n=36)$ & $\mathbf{P}$ \\
\hline \multicolumn{5}{|l|}{ Clinical } \\
\hline Age [years] & $81.6 \pm 6.9$ & $81.3 \pm 7.9$ & $81.8 \pm 5.8$ & 0.7 \\
\hline Gender ( $\%$ female) & $41(57 \%)$ & $20(54 \%)$ & $19(56 \%)$ & 0.9 \\
\hline Body surface area $\left[\mathrm{m}^{2}\right]$ & $1.8 \pm 0.21$ & $1.79 \pm 0.21$ & $1.81 \pm 0.22$ & 0.7 \\
\hline Hypertension & $56(80 \%)$ & $29(85 \%)$ & $27(74 \%)$ & 0.4 \\
\hline Diabetes mellitus & $32(45 \%)$ & $16(47 \%)$ & $16(43 \%)$ & 0.7 \\
\hline Coronary artery disease & $35(49 \%)$ & $18(53 \%)$ & $17(46 \%)$ & 0.55 \\
\hline \multicolumn{5}{|l|}{ Echocardiography } \\
\hline LVEDD [cm] & $4.5 \pm 0.6$ & $4.5 \pm 0.6$ & $4.5 \pm 0.6$ & 0.8 \\
\hline LVMI $\left[\mathrm{g} / \mathrm{m}^{2}\right]$ & $133 \pm 49$ & $136 \pm 58$ & $128 \pm 35.6$ & 0.6 \\
\hline LVEF [\%] & $61.0 \pm 4.8$ & $61 \pm 5.3$ & $61 \pm 4.4$ & 0.96 \\
\hline LVOT area $\left[\mathrm{cm}^{2}\right]$ & $3.3 \pm 0.5$ & $3.4 \pm 0.5$ & $3.2 \pm 0.46$ & 0.2 \\
\hline LVOT VTI [cm] & $22.8 \pm 4.6$ & $24.5 \pm 4.2$ & $21.3 \pm 4.4$ & 0.005 \\
\hline AV VTI [cm] & $97.3 \pm 20.3$ & $113 \pm 14.1$ & $82.2 \pm 12$ & $<0.0001$ \\
\hline Mean gradient $[\mathrm{mmHg}]$ & $41 \pm 14$ & $52 \pm 11.0$ & $29.5 \pm 5.5$ & $<0.0001$ \\
\hline AVA $\left[\mathrm{cm}^{2}\right]$ & $0.77 \pm 0.17$ & $0.71 \pm 0.17$ & $0.82 \pm 0.14$ & 0.002 \\
\hline Stroke volume index $\left[\mathrm{mL} / \mathrm{m}^{2}\right]$ & $42 \pm 10.4$ & $46.1 \pm 9.8$ & $38.2 \pm 9.7$ & 0.001 \\
\hline \multicolumn{5}{|l|}{ Invasive hemodynamic } \\
\hline Mean gradient $[\mathrm{mmHg}]$ & $45.9 \pm 13.4$ & $51.3 \pm 13$ & $40.3 \pm 11.6$ & 0.0003 \\
\hline $\mathrm{AVA}\left[\mathrm{cm}^{2}\right]$ & $0.68 \pm 0.21$ & $0.65 \pm 0.17$ & $0.66 \pm 0.9$ & 0.9 \\
\hline \multicolumn{5}{|l|}{ Computed tomography } \\
\hline LVOT area $\left[\mathrm{cm}^{2}\right]$ & $4.24 \pm 1.12$ & $4.3 \pm 1.1$ & $4.2 \pm 1.1$ & 0.6 \\
\hline Ellipticity index & $1.27 \pm 0.09$ & $1.25 \pm 0.08$ & $1.27 \pm 0.09$ & 0.4 \\
\hline Calcium score $[\mathrm{AU}]$ & $2347 \pm 1196$ & $2678 \pm 1151$ & $1984 \pm 1155$ & 0.02 \\
\hline
\end{tabular}

AV - aortic valve; AVA — aortic valve area; LVEDD — left ventricular end diastolic diameter; LVEF — left ventricular ejection fraction; LVMI — left ventricular mass index; LVOT — left ventricular outflow tract; VTI — velocity time integra

lar. CaSc tended to be lower, implying less severe valvular disease. The prevalence of SV index $\leq 35 \mathrm{~mL} / \mathrm{m}^{2}$ was similar between TD and FD $(\mathrm{p}=0.8)$.

There was no difference between non-invasively and invasively determined mean gradients in echocardiographically TC patients (as well as $\mathrm{TD})$, nor was there a difference in invasive mean gradients between TC and FD (Fig. 2A). While echocardiographic AVA was slightly smaller in $\mathrm{TC}$ when compared to TD and FD, there were no significant differences in mean invasive AVA across groups (Fig. 2B).

After a thorough review of the echocardiographic examinations of the $17 \mathrm{FD}$ we found that a reliable right parasternal view was missing in 6 patients, LVOT VTI tracings were suboptimal in 3 patients, and inadequate due to poor imaging quality in 1 case. Seven of the 17 patients had an echocardiographic mean gradient $>35 \mathrm{mmHg}$, $5 \mathrm{had}$ a mean gradient $>30 \mathrm{mmHg}$, and only 5 of them had a gradient between 25 and $29 \mathrm{mmHg}$.

Calcium score levels had a positive predictive value of $95 \%$ for the correct diagnosis of severe AS (likely) in concordants and of $93 \%$ in discordants.

\section{Discussion}

The main findings of this study are as follows: 1) In a selected group of symptomatic patients with severe AS and discordant echocardiographic findings (with a mean gradient $>30 \mathrm{mmHg}$ ), who underwent full hemodynamic evaluation, technical errors leading to underestimation of the true aortic gradient (pseudo-discordance) are almost as common as true low flow low gradient severe AS; 2) CT assessment of LVOT area was not helpful in differentiating between true and false discord- 
Table 2. Baseline clinical, echocardiographic, invasive hemodynamic, and computed tomography characteristics.

\begin{tabular}{|c|c|c|c|c|c|c|}
\hline & $\begin{array}{c}\text { False } \\
\text { discordant } \\
(\mathbf{n}=17)\end{array}$ & $\begin{array}{c}\text { True } \\
\text { discordant } \\
\text { (n=19) }\end{array}$ & $\begin{array}{c}\text { True } \\
\text { concordant } \\
(\mathrm{n}=34)\end{array}$ & $P^{*}$ & $P^{* *}$ & $P^{* * *}$ \\
\hline \multicolumn{7}{|l|}{ Clinical } \\
\hline Age [years] & $82.8 \pm 4.5$ & $81.4 \pm 6.6$ & $81.7 \pm 7.9$ & 0.8 & 0.5 & 0.4 \\
\hline Gender ( $\%$ female) & $6(37.5 \%)$ & $14(74 \%)$ & $18(53 \%)$ & 0.02 & 0.37 & 0.05 \\
\hline Body surface area $\left[\mathrm{m}^{2}\right]$ & $1.84 \pm 0.22$ & $1.80 \pm 0.23$ & $1.79 \pm 0.21$ & 0.9 & 0.5 & 0.6 \\
\hline Hypertension & $12(69 \%)$ & $15(79 \%)$ & $29(85 \%)$ & 0.8 & 0.4 & 0.84 \\
\hline Diabetes mellitus & $8(44 \%)$ & $8(42 \%)$ & $16(47 \%)$ & 1 & 0.8 & 1 \\
\hline Coronary artery disease & $10(56 \%)$ & $7(37 \%)$ & $18(53 \%)$ & 0.4 & 0.9 & 0.3 \\
\hline \multicolumn{7}{|l|}{ Echocardiography } \\
\hline LVEDD [cm] & $4.5 \pm 0.5$ & $4.6 \pm 0.6$ & $4.5 \pm 0.6$ & 0.7 & 0.9 & 0.6 \\
\hline LVMI $\left[\mathrm{g} / \mathrm{m}^{2}\right]$ & $132 \pm 35$ & $128 \pm 40$ & $138 \pm 60$ & 0.5 & 0.7 & 0.7 \\
\hline LVEF [\%] & $62 \pm 4$ & $59.7 \pm 4.2$ & $61 \pm 5.4$ & 0.4 & 0.46 & 0.09 \\
\hline LVOT area $\left[\mathrm{cm}^{2}\right]$ & $3.3 \pm 0.4$ & $3.1 \pm 0.5$ & $3.3 \pm 0.5$ & 0.25 & 0.8 & 0.38 \\
\hline LVOT VTI [cm] & $21.7 \pm 43$ & $21.2 \pm 4.5$ & $24.3 \pm 4.3$ & 0.02 & 0.05 & 0.8 \\
\hline AV VTI [cm] & $84.8 \pm 10.7$ & $80.6 \pm 12.5$ & $113 \pm 14.4$ & $<0.0001<$ & $<0.0001$ & 0.3 \\
\hline Mean $\Delta[\mathrm{mmHg}]$ & $32 \pm 6$ & $27.5 \pm 4.9$ & $51.8 \pm 11.5$ & $<0.0001$ & $<0.0001$ & 0.01 \\
\hline AVA $\left[\mathrm{cm}^{2}\right]$ & $0.85 \pm 0.14$ & $0.83 \pm 0.15$ & $0.71 \pm 0.17$ & 0.006 & 0.004 & 0.8 \\
\hline Stroke volume index $\left[\mathrm{mL} / \mathrm{m}^{2}\right]$ & $39.2 \pm 8.5$ & $37.8 \pm 102$ & $45.9 \pm 10$ & 0.008 & 0.02 & 0.63 \\
\hline \multicolumn{7}{|l|}{ Invasive hemodynamic } \\
\hline Mean $\Delta[\mathrm{mmHg}]$ & $51 \pm 6.6$ & $31.9 \pm 6.4$ & $52.2 \pm 12.9$ & $<0.0001$ & 0.5 & $<0.0001$ \\
\hline $\mathrm{AVA}\left[\mathrm{cm}^{2}\right]$ & $0.66 \pm 0.25$ & $0.69 \pm 0.19$ & $0.65 \pm 0.17$ & 0.4 & 0.9 & 0.6 \\
\hline \multicolumn{7}{|l|}{ Computed tomography } \\
\hline LVOT area $\left[\mathrm{cm}^{2}\right]$ & $4.4 \pm 1.4$ & $4.11 \pm 0.9$ & $4.2 .9 \pm 0.8$ & 0.5 & 0.8 & 0.5 \\
\hline Ellipticity index & $1.27 \pm 0.11$ & $1.26 \pm 0.08$ & $1.2 \pm 0.08$ & 0.6 & 0.5 & 0.5 \\
\hline Calcium score [AU] & $2369 \pm 1076$ & $1707 \pm 1141$ & $2724 \pm 1159$ & 0.005 & 0.3 & 0.1 \\
\hline
\end{tabular}

All abbreviations as in the main text and in Table $1 ;{ }^{*}$ Compares concordants and true discordants; ${ }^{*}$ Compares concordants and false discordants; ${ }^{* *}$ Compares true discordants and false discordants

ance; 3) Without an adequate transvalvular velocity recording from all echocardiographic windows the diagnosis of low gradient severe AS cannot be definitively established, and CT determination of the CaSc should be mandatory (Fig. 3).

These findings are in full agreement with the current guidelines stating that in patients older than 70 years, who have typical symptoms, AVA $<0.8 \mathrm{~cm}^{2}$, and a high $\mathrm{CaSc}$ ( $>1200 \mathrm{AU}$ in women, and $>2000$ AU in men, respectively) are associated with a very high probability of true severe AS $[1,2]$. The findings have important implications for the diagnosis of true paradoxical low flow low gradient severe AS. Establishing its presence echocardiographically remains an exclusion diagnosis and is confounded by several factors that may lead to spuriously discordant findings.
Underestimation of peak velocity and Doppler-derived gradients. In many cases, heavily calcified valves may not allow the maximal envelope velocity to be obtained from an apical window, even when the angle alignment is optimal and the recorded signal appears to be of diagnostic quality. The use of multiple interrogation windows is paramount - in particular, the use of a right (or suprasternal) window, which allows sampling the velocity of the approaching jet without interposition of a calcified valve that may filter out the maximum signal. Previous studies have shown that relying solely on the apical view may lead to a significant underestimation of the peak and mean gradients in between $20 \%$ and $50 \%$ of cases [11, 12].

Underestimation of AVA by the continuity equation. In clinical practice, application of the 


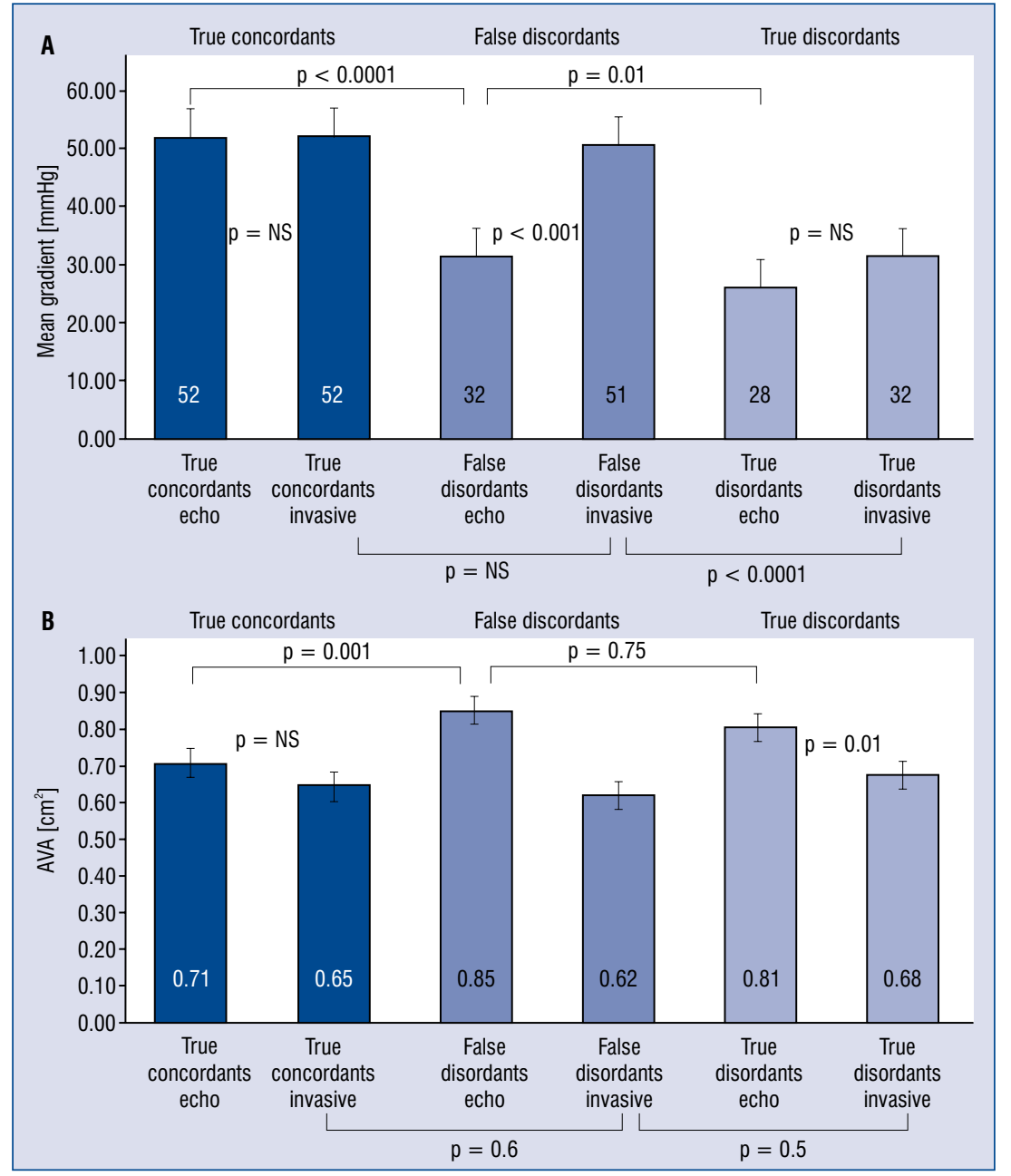

Figure 2. All patients with aortic valve area $(A V A) \leq 1 \mathrm{~cm}^{2}(\mathrm{n}=70) ;$ A. Mean gradient $[\mathrm{mmHg}] ;$ B. $A V A\left[\mathrm{~cm}^{2}\right]$.

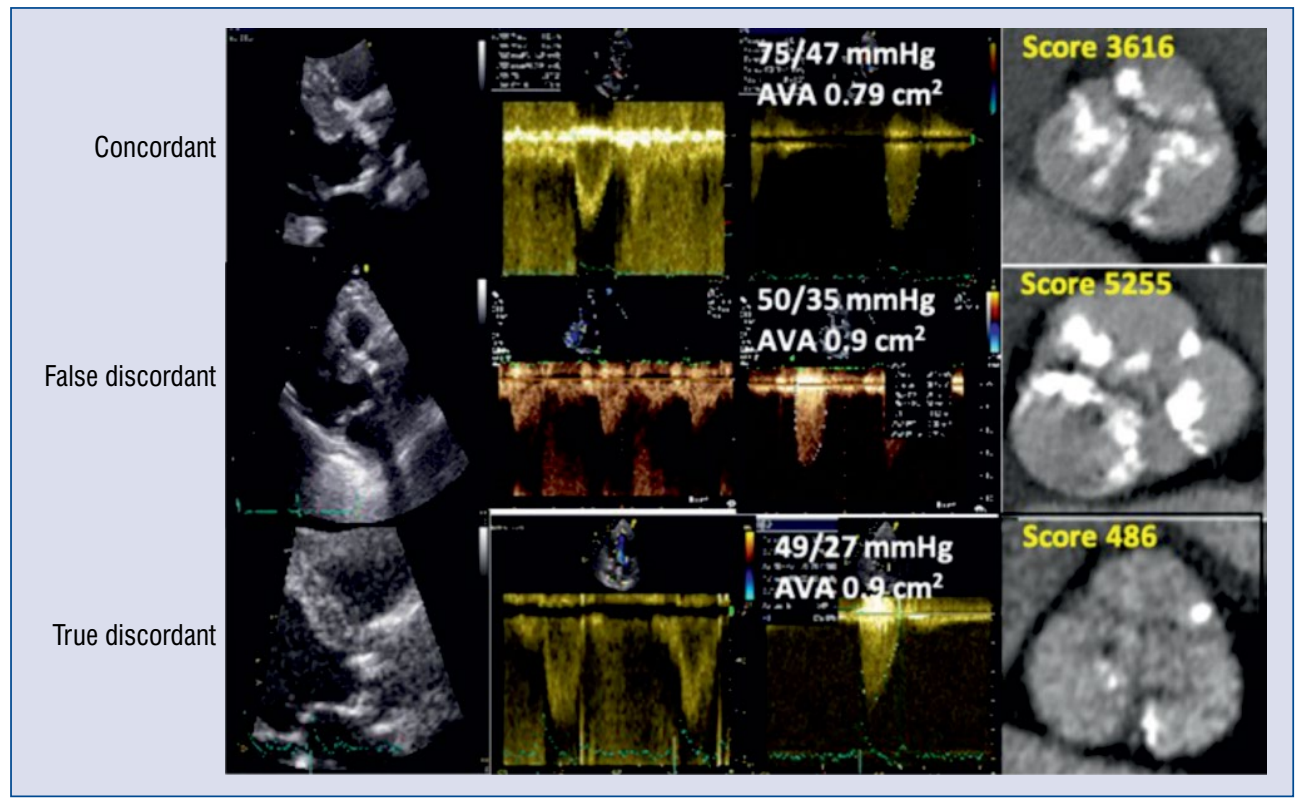

Figure 3. Three typical patients; AVA — aortic valve area. 
continuity equation relies on calculation of the LVOT area by a single diameter assuming circularity. However, the LVOT area has been shown to be elliptic by both echocardiography [4] and CT studies $[5,13]$, so underestimation of the true valve area is possible. We therefore assessed the anatomic LVOT area in the three-dimensional CT data set.

Small body size. Patients with small body size may show valve areas $\leq 1.0 \mathrm{~cm}^{2}$ even with non-severe AS, and they have smaller than expected gradients due to a lower cardiac output, which simply reflects their smaller perfused muscle mass.

We sought to determine the distribution of the root causes by first validating the hemodynamic severity of the lesion by cardiac catheterization, and then by assessing the lesion severity by an additional imaging modality independent of both echocardiography and cardiac catheterization $(\mathrm{CaSc})$, and finally by determining the true LVOT area by CT to assess the potential impact of the circularity assumption for the determination of AVA in each patient individually [14].

Contrary to our expectation, underestimation of the gradients by echocardiography was the most common cause of misclassification. Even though all the echocardiographic studies were performed in the high-volume laboratory of an academic tertiary referral center by experienced echocardiography technologists using state-of-the-art equipment, Doppler acquisition from the right parasternal view was unreliable in six patients, and Doppler tracings were retrospectively identified to be of suboptimal quality in another 4, explaining the misclassification in $59 \%$ of the FD patients. The fact that valve calcification, potentially obscuring the maximum velocity signals from an apical window, tended to be particularly high in patients in whom gradients were underestimated (Fig. 3) further supports this assessment. The main reason for this finding is probably related to the nature of the studied patient population, which consisted of elderly and highly symptomatic individuals, many of whom had multiple comorbidities and had difficulty in changing body position during the echocardiographic examination. This, however, is typical for the patient population routinely referred for echocardiographic evaluation of valvular heart disease to date; hence, the findings are of wider clinical relevance. Even though underestimation of the LVOT diameter is considered to be responsible for most of the AVA underestimation encountered in clinical practice [4-6], a CT assessment of the LVOT area did not help in differentiating between concordants and discordants in this study. As expected, the LVOT area assessed by CT was elliptical and larger than the echocardiographically estimated LVOT area. However, because the LVOT was consistently found to be elliptical in all patients, with a similar distribution across the diverse sub-groups, the eccentricity index did not facilitate distinguishing between TD and FD.

\section{Comparison with previous studies}

Discordance in echocardiographic measures of severe AS (velocity, gradient, and valve area) has been reported in up to $40 \%$ of patients, the most common constellation encountered in clinical practice being a low mean gradient $(<40 \mathrm{mmHg})$ despite a small AVA $\left(\leq 1 \mathrm{~cm}^{2}\right)$ [15-17]. Several studies attempted to elucidate the clinical importance of these findings by serial echocardiographic and clinical follow-up:

Maes el al. [18] studied 205 patients with a working diagnosis of "paradoxical low flow low gradient severe AS". Eighty-two percent increased their gradient during follow up and 50\% progressed to high-gradient severe AS [18]. Among the 1131 patients evaluated in this study, only 34 were excluded due to poor image quality. Tribouilloy retrospectively evaluated the echocardiograms of 59 patients who were followed up with serial echocardiograms during 2 years [19]. No patient was excluded for poor imaging quality. Mean Doppler gradient increased in $82 \%$ of these patients during follow-up, fulfilling the criteria of severe high gradient AS in $41 \%$. Zusman et al. [20] retrospectively evaluated a group of 303 patients with symptomatic normal-flow low-gradient severe AS and concluded that these patients may benefit from intervention when compared to clinical treatment. No patient was excluded based on imaging quality. Only 61 patients had a second echocardiographic examination, and 13 of those showed increased gradients [20]. In a similar study Kang et al. [21] evaluated a group of 284 patients with normal flow low gradient severe AS, of whom 186 were followed clinically. Again, no patient was excluded due to poor imaging. Of note, 145 of the 186 patients followed up clinically increased their gradients on subsequent echocardiographic examinations [21].

The observed increase in gradients during serial echocardiographic examinations in these studies is compatible with the hypothesis that in many patients low flow low gradient AS with normal LV function may represent an intermediate stage of AS, between moderate and high gradient AS, rather than a more advanced stage of the disease [19], 
a conclusion further supported by the study by Slimani et al. [22], which demonstrated that patients with paradoxical low flow low gradient severe AS less frequently display reduced longitudinal deformation, LV hypertrophy, or myocardial fibrosis than patients with high gradient severe AS [22].

The most conclusive and practical approach to the diagnostic conundrum of discordant echocardiographic findings appears to be assessment of the aortic valve CaSc by CT. This is compatible with the findings by Shen et al. [23], who evaluated the effects of age and $\mathrm{AV}$ anatomy on the relationship between AV calcification and the echocardiographic parameters of AS severity in 200 patients with severe AS and preserved LV function. The authors concluded that in elderly patients AV calcification appears to be the main factor significantly associated with the severity of AS, and CaSc evaluation should be used for the differential diagnosis of severe AS with discordant echocardiographic findings [23]. The results of the present study expand their findings by first comparing echocardiographic results to invasive measurements (to discriminate between true and false discordant findings) and then by evaluating LVOT anatomy and degree of valve calcification quantitatively per CT across patient groups, enabling a proper root cause analysis of this relationship.

Finally, current guidelines [1] recommend AV replacement in patients with symptomatic severe AS or with LVEF $<50 \%$, while intervention is not indicated in patients with symptomatic moderate AS and LV dysfunction [24]. In order to clarify whether a more aggressive approach is necessary in these patients, the Transcatheter Aortic Valve Replacement to UNload the Left ventricle in patients with $\mathrm{AD}$ vanced heart failure (TAVR UNLOAD) trial aims to randomize 300 patients into two arms: transcatheter aortic valve replacement combined with optimized heart failure therapy versus optimal heart failure therapy alone [25]. The primary endpoint will be a composite of all-cause death, disabling stroke, heart failure hospitalizations, symptomatic AV disease, or non-disabling stroke.

\section{Limitations of the study}

The main limitations of this study are the highly selected population, limited sample size, and the fact that the echocardiographic and invasive studies were not performed simultaneously. However, such simultaneous recordings are unlikely to have improved agreement between invasive and non-invasive data because the time difference between the studies was not long (79 \pm 70 days), and obtaining an adequate right parasternal window uniformly requires patients to lie fully turned to the right, which is not practical during cardiac catheterization. In addition, the study group consisted exclusively of symptomatic patients, increasing the pre-test probability for severe AS. However, this is the group of clinical interest because asymptomatic patients rarely undergo invasive hemodynamic investigations.

Finally, the inclusion of all consecutively studied patients in this investigation, without retrospective exclusion of patients with more challenging signal quality, should not be seen as a weakness but as a strength of the study. It allowed us to reliably analyze the true root causes of discordant findings in routine echocardiography. Such information is important to overcome selection bias, which may lead to underestimation of pseudo-discordance in clinical practice.

\section{Conclusions}

Discordant echocardiographic findings are commonly found in patients with symptomatic AS. In patients with pseudo-discordance underestimation of the true mean gradient due to technical difficulties is an important root cause for these discrepant findings. LVOT area by echocardiography or CT cannot differentiate between TD and FD. Low gradient severe AS can only be diagnosed when a reliable Doppler recording from all echocardiographic windows is available. Otherwise, a CaSc determination by computerized tomography is required.

\section{Conflict of interest: None declared}

\section{References}

1. Otto CM, Nishimura RA, Bonow RO, et al. 2020 AHA/ACC guideline for the management of patients with valvular heart disease: executive summary: a report of the American College of Cardiology/American Heart Association Task Force on Practice Guidelines. J Am Coll Cardiol. 2021; 77(4): e25-97, doi: 10.1016/j.jacc.2020.11.018, indexed in Pubmed: 33342586.

2. Baumgartner H, Falk V, Bax JJ, et al. ESC Scientific Document Group, ESC Scientific Document Group. 2017 ESC/EACTS Guidelines for the management of valvular heart disease. Eur Heart J. 2017; 38(36): 2739-2791, doi: 10.1093/eurheartj/ehx391, indexed in Pubmed: 28886619.

3. Pibarot P, Dumesnil JG. Aortic stenosis suspected to be severe despite low gradients. Circ Cardiovasc Imaging. 2014; 7(3): 545-551, doi: 10.1161/CIRCIMAGING.113.001375, indexed in Pubmed: 24847008.

4. Baumgartner H, Kratzer H, Helmreich G, et al. Determination of aortic valve area by Doppler echocardiography using the continuity equation: a critical evaluation. Cardiology. 1990; 77(2): 101-111, doi: 10.1159/000174590, indexed in Pubmed: 2397487. 
5. Tops LF, Wood DA, Delgado V, et al. Noninvasive evaluation of the aortic root with multislice computed tomography implications for transcatheter aortic valve replacement. JACC Cardiovasc Imaging. 2008; 1(3): 321-330, doi: 10.1016/j.jcmg.2007.12.006, indexed in Pubmed: 19356444.

6. Chin CWL, Khaw HJ, Luo E, et al. Echocardiography underestimates stroke volume and aortic valve area: implications for patients with small-area low-gradient aortic stenosis. Can J Cardiol. 2014; 30(9): 1064-1072, doi: 10.1016/j.cjca.2014.04.021, indexed in Pubmed: 25151288.

7. Baumgartner H, Hung J, Bermejo J, et al. Recommendations on the Echocardiographic Assessment of Aortic Valve Stenosis: A Focused Update from the European Association of Cardiovascular Imaging and the American Society of Echocardiography. J Am Soc Echocardiogr. 2017; 30(4): 372-392, doi: 10.1016/j. echo.2017.02.009, indexed in Pubmed: 28385280.

8. Budoff MJ, Nasir K, Kinney GL, et al. Coronary artery and thoracic calcium on noncontrast thoracic $\mathrm{CT}$ scans: comparison of ungated and gated examinations in patients from the COPD Gene cohort. J Cardiovasc Comput Tomogr. 2011; 5(2): 113-118, doi: 10.1016/j.jcct.2010.11.002, indexed in Pubmed: 21167806.

9. Agatston A, Janowitz W, Hildner F, et al. Quantification of coronary artery calcium using ultrafast computed tomography. J Am Coll Cardiol. 1990; 15(4): 827-832, doi: 10.1016/07351097(90)90282-t.

10. Takasu J, Katz R, Nasir K, et al. Relationships of thoracic aortic wall calcification to cardiovascular risk factors: the Multi-Ethnic Study of Atherosclerosis (MESA). Am Heart J. 2008; 155(4): 765-771, doi: 10.1016/j.ahj.2007.11.019, indexed in Pubmed: 18371491.

11. Thaden JJ, Nkomo VT, Lee KJe, et al. Doppler imaging in aortic stenosis: the importance of the nonapical imaging windows to determine severity in a contemporary cohort. J Am Soc Echocardiogr. 2015; 28(7): 780-785, doi: 10.1016/j.echo.2015.02.016, indexed in Pubmed: 25857547.

12. de Monchy CC, Lepage L, Boutron I, et al. Usefulness of the right parasternal view and non-imaging continuous-wave Doppler transducer for the evaluation of the severity of aortic stenosis in the modern area. Eur J Echocardiogr. 2009; 10(3): 420-424, doi: 10.1093/ejechocard/jen301, indexed in Pubmed: 19036750.

13. Hamdan A, Guetta V, Konen E, et al. Deformation dynamics and mechanical properties of the aortic annulus by 4-dimensional computed tomography: insights into the functional anatomy of the aortic valve complex and implications for transcatheter aortic valve therapy. J Am Coll Cardiol. 2012; 59(2): 119-127, doi: 10.1016/j.jacc.2011.09.045, indexed in Pubmed: 22222074.

14. Messika-Zeitoun D, Oh JK, Topilsky Y, et al. Low-gradient aortic stenosis: solving the conundrum using multi-modality imaging. Prog Cardiovasc Dis. 2018; 61(5-6): 416-422, doi: 10.1016/j. pcad.2018.11.006, indexed in Pubmed: 30445161.
15. Berthelot-Richer M, Pibarot P, Capoulade R, et al. Discordant grading of aortic stenosis severity: echocardiographic predictors of survival benefit associated with aortic valve replacement. JACC Cardiovasc Imaging. 2016; 9(7): 797-805, doi: 10.1016/j. jcmg.2015.09.026, indexed in Pubmed: 27209111.

16. Minners J, Allgeier M, Gohlke-Baerwolf C, et al. Inconsistent grading of aortic valve stenosis by current guidelines: haemodynamic studies in patients with apparently normal left ventricular function. Heart. 2010; 96(18): 1463-1468, doi: 10.1136/ hrt.2009.181982, indexed in Pubmed: 20813727.

17. Hachicha Z, Dumesnil JG, Bogaty P, et al. Paradoxical low-flow, low-gradient severe aortic stenosis despite preserved ejection fraction is associated with higher afterload and reduced survival. Circulation. 2007; 115(22): 2856-2864, doi: 10.1161/CIRCULATIONAHA.106.668681, indexed in Pubmed: 17533183.

18. Maes F, Boulif J, Piérard S, et al. Natural history of paradoxical low-gradient severe aortic stenosis. Circ Cardiovasc Imaging. 2014; 7(4): 714-722, doi: 10.1161/CIRCIMAGING.113.001695, indexed in Pubmed: 24777938.

19. Tribouilloy C, Rusinaru D, Charles V, et al. Progression of low-gradient, low-flow, severe aortic stenosis with preserved left ventricular ejection fraction. Am J Cardiol. 2015; 116(4): 612617, doi: 10.1016/j.amjcard.2015.05.023.

20. Zusman O, Pressman GS, Banai S, et al. Intervention versus observation in symptomatic patients with normal flow low gradient severe aortic stenosis. JACC Cardiovasc Imaging. 2018; 11(9): 1225-1232, doi: 10.1016/j.jcmg.2017.07.020, indexed in Pubmed: 29055632.

21. Kang DH, Jang JY, Park SJ, et al. Watchful observation versus early aortic valve replacement for symptomatic patients with normal flow, low-gradient severe aortic stenosis. Heart. 2015; 101(17): 1375-1381, doi: 10.1136/heartjnl-2015-307528, indexed in Pubmed: 26105038.

22. Slimani A, Roy C, de Meester C, et al. Structural and functional correlates of gradient-area patterns in severe aortic stenosis and normal ejection fraction. JACC Cardiovasc Imaging. 2021; 14(3): 525-536, doi: 10.1016/j.jcmg.2020.09.031, indexed in Pubmed: 33221240.

23. Shen M, Tastet L, Capoulade R, et al. Effect of age and aortic valve anatomy on calcification and haemodynamic severity of aortic stenosis. Heart. 2017; 103(1): 32-39, doi: 10.1136/ heartjnl-2016-309665, indexed in Pubmed: 27504001.

24. Strange G, Stewart S, Celermajer D, et al. Poor long-term survival in patients with moderate aortic stenosis. J Am Coll Cardiol. 2019; 74(15): 1851-1863, doi: 10.1016/j.jacc.2019.08.004, indexed in Pubmed: 31491546.

25. Spitzer E, Mieghem NV, Pibarot P, et al. Rationale and design of the Transcatheter Aortic Valve Replacement to UNload the Left ventricle in patients with ADvanced heart failure (TAVR UNLOAD) trial. Am Heart J. 2016; 182: 80-88, doi: 10.1016/j. ahj.2016.08.009. 